\title{
A UNIVERSAL MODEL FOR DYNAMICAL SYSTEMS WITH QUASI-DISCRETE SPECTRUM
}

\author{
BY JAMES R. BROWN
}

Communicated by Paul Halmos, March 25, 1969

Let $K_{d}$ denote the circle group (unit interval with addition mod 1) with the discrete topology, let $\hat{K}_{d}$ be its dual (the Bohr compactification of the integers), and let $\hat{K}_{d}^{\omega}$ denote the direct product of countably many copies of $\hat{K}_{d}$. We define an affine transformation $\hat{\phi}$ of the group $\hat{K}_{d}^{\omega}$ by

$$
\hat{\phi}\left(x_{1}, x_{2}, x_{3}, \cdots\right)=\left(x_{1}+\hat{a}, x_{1}+x_{2}, x_{2}+x_{3}, \cdots\right),
$$

where $a \in \hat{K}_{d}$ is defined by

$$
\hat{a}(t)=t \quad\left(t \in K_{d}\right) .
$$

Implicit in the proof of the representation theorem of Halmos and von Neumann [5] for measure-preserving transformations with discrete spectrum is the fact that every such system is a factor (homomorphic image) of the translation $x \rightarrow x+\hat{a}$ on $\hat{K}_{d}$. Moreover, it follows easily from the fact that any subgroup $\Gamma$ of $K_{d}$ is the union of its finitely generated subgroups (as is any group) that any system with discrete spectrum is an inverse limit of direct products of ergodic translations on the circle group $K$ (usual topology) and cyclic permutations of finite sets. A discussion of such inverse limits is given in [2]. In the present note we extend these two results to the ergodic dynamical systems $\Phi$ with quasi-discrete spectrum that were introduced by L. M. Abramov [1] in the measure-theoretic case and by F. Hahn and W. Parry [3] in the topological case.

In each case, by virtue of the respective representation theorems, we can assume without loss of generality that the system $\Phi=(G, B$, $\mu, \phi)$ is such that $G$ is a compact abelian group, $B$ is the class of Baire subsets of $G, \mu$ is normalized Haar measure, and $\phi$ is an ergodic, continuous, affine transformation of $G$ with quasi-discrete spectrum [1], [3], [4].

By an affine transformation of $G$ we mean a map $\phi: G \rightarrow G$ of the form $\phi(x)=\tau(x)+a$, where $\tau$ is an automorphism of $G$ and $a \in G$. Let us denote by $\sigma$ the endomorphism of $G$ defined by $\sigma(x)=\tau(x)-x$. Then $\phi$ is said to have quasi-discrete spectrum provided that

$$
\bigcap_{n=1}^{\infty} \sigma^{n} G=(0)
$$


THEOREM 1. Every ergodic dynamical system with quasi-discrete spectrum is a factor of the system $\hat{\Phi}=\left(\hat{K}_{d}^{\omega}, \hat{\otimes}, \hat{\mu}, \hat{\phi}\right)$, where $\hat{\mu}$ is the Haar measure on the class $\hat{B}$ of Baire subsets of $\hat{K}_{d}^{\omega}$.

REMARK. This theorem, as well as Theorem 2 below, can be read in any one of three categories-affine transformations on compact abelian groups with continuous homomorphisms for the maps, classical dynamical systems with continuous maps, or abstract (measuretheoretic) systems with measure-preserving maps. In each case the theorem asserts the existence of a map $\psi$ of $\hat{K}_{d}^{\omega}$ onto $G$ such that $\psi \hat{\phi}=\phi \psi$. We indicate this by $\psi: \hat{\Phi} \rightarrow \Phi$. We prove these theorems in the first category indicated, their validity in the other two following from the representation theorems of [1] and [3].

Proof. We define the homomorphism $\psi$ by describing its adjoint $\psi^{*}: \Gamma \rightarrow K_{d}^{\omega}$, where $K_{d}^{\omega}$, the direct sum of countably many copies of $K_{d}$, is the dual of $\hat{K}_{d}^{\omega}$, and $\Gamma=\hat{G}$ is the dual of $G$. Specifically, we set

$$
\psi^{*}(\gamma)=\left(\gamma(a), \gamma(\sigma a), \gamma\left(\sigma^{2} a\right), \cdots\right) .
$$

We need to show that $\psi^{*}$ is a monomorphism, and that $\psi \hat{\phi}=\phi \psi$. The latter is equivalent, after observing that $\psi(\hat{a}, 0,0, \cdots)=a$, to the condition $\psi \hat{\sigma}=\sigma \psi$, where

$$
\hat{\sigma}\left(x_{1}, x_{2}, x_{3}, \cdots\right)=\left(0, x_{1}, x_{2}, \cdots\right) \text {. }
$$

This condition, in turn, is equivalent to $\hat{\sigma}^{*} \psi^{*}=\psi^{*} \sigma^{*}$, which follows easily from (1). Since $\psi^{*}$ is readily seen to be a homomorphism, the proof reduces to showing that it is one-to-one.

Let us denote by $\Gamma_{n}$ the annihilator of $\sigma^{n} G$. Then $\Gamma_{n}$ is the kernel of $\sigma^{*_{n}}$ and $\Gamma=\bigcup_{n=1}^{\infty} \Gamma_{n}$. Suppose $\gamma \in \Gamma_{n+1}$ and $\psi^{*}(\gamma)=0$, i.e. $\gamma\left(\sigma^{k} a\right)=0$ for each $k=1,2, \cdots, n$ and $\gamma\left(\sigma^{k} x\right)=0$ for all $x \in G$ and $k>n$. Let $g=\sigma^{* n} \gamma$. Then

$$
\begin{aligned}
g(\phi x) & =\gamma\left(\sigma^{n}(x+\sigma x+a)\right) \\
& =\gamma\left(\sigma^{n} x\right)+\gamma\left(\sigma^{n+1} x\right)+\gamma\left(\sigma^{n} a\right) \\
& =g(x) .
\end{aligned}
$$

By ergodicity it follows that $g$ is a constant, necessarily 0 . Thus $\gamma \in \Gamma_{n}$. By a repetition of this argument, it follows that $\gamma=0$, i.e. the kernel of $\psi^{*}$ is trivial.

Note that the mapping $\psi^{*}$ is an embedding of the quasi-eigenvalue system of $\Phi$ (as defined in [3]) into that of $\hat{\Phi}$. Let us call a group $H \subseteq K_{d}^{\omega}$ shift-invariant if $\hat{\sigma}^{*} H \subseteq H$.

COROLLARY. If $\Phi$ is any ergodic dynamical system, then its system 
of quasi-eigenvalues is isomorphic to a shift-invariant subgroup of $K_{d}^{\omega}$. Conversely, any such subgroup is the quasi-eigenvalue system of some ergodic dynamical system with quasi-discrete spectrum.

Suppose that $\gamma^{1}, \gamma^{2}, \cdots, \gamma^{k} \in K_{d}^{\omega}$, and let $\Gamma$ be the smallest shiftinvariant group containing them. Then $\Gamma \subseteq K_{d}^{n}$, the subgroup of elements $\gamma \in K_{d}^{\omega}$ with at most the first $n$ components different from 0 , for some $n$. Moreover, $\Gamma \subseteq \Gamma^{1} \oplus \Gamma^{1} \oplus \cdots \oplus \Gamma^{1} \oplus(0)$ ( $n$ factors) for some finitely generated subgroup $\Gamma^{1}$ of $K_{d}$. (Let $\Gamma^{1}$ be generated by $\gamma_{i}^{\prime}, i=1, \cdots, n ; j=1, \cdots, k$.) Since $\Gamma^{1}$ is a direct sum of cyclic groups, we obtain a decomposition of the above sum into the quasieigenvalue systems of some special dynamical systems to be described now.

We shall call the dynamical system $\Phi_{\alpha}=\left(G_{\alpha}, \Theta_{\alpha}, \mu_{\alpha}, \phi_{\alpha}\right)$ special if $\mu_{\alpha}$ is Haar measure on the Baire sets $B_{\alpha}$ of $G_{\alpha}$, where either $\alpha \in K$ and $G_{\alpha}=K^{n}$ or $\alpha \in Z_{t}$ (the additive group of integers $\bmod t$ ) and $G_{\alpha}=Z_{t}^{n}$, and if

$$
\phi_{\alpha}\left(x_{1}, \cdots, x_{n}\right)=\left(x_{1}+\alpha, x_{1}+x_{2}, \cdots, x_{n-1}+x_{n}\right) .
$$

In the first case, it is assumed that $\alpha$ is irrational, and in the second that the order of $\alpha$ is $t$. Thus $\Phi_{\alpha}$ is ergodic. Its system of quasieigenvalues is $(\alpha) \oplus(\alpha) \oplus \cdots \oplus(\alpha) \oplus(0)$. Such systems were considered in [3] under the name of "diagonal" transformations.

THEOREM 2. $\hat{\Phi}$ is an inverse limit of products of special systems. More generally, any dynamical system with quasi-discrete spectrum is an inverse limit of factors of products of special systems.

Clearly, those familiar properties (see [1], [3], [4]) of dynamical systems with quasi-discrete spectrum, such as zero entropy, that are preserved under the formation of products, factors and inverse limits follow readily from Theorems 1 and 2 .

\section{BIBLIOGRAPHY}

1. L. M. Abramov, Metric automorphisms with quasi-discrete spectrum, Izv. Akad. Nauk SSSR Ser. Mat. 26 (1962), 513-530; English transl., Amer. Math. Soc. Transl., (2) 39 (1964), 37-56.

2. J. R. Brown, Inverse limits, entropy, and weak isomorphism for discrete dynamical systems (to appear).

3. F. Hahn and W. Parry, Minimal dynamical systems with quasi-discrete spectrum, J. London Math. Soc. 40 (1965), 309-323.

4. - Some characteristic properties of dynamical systems with quasi-discrete spectra, Math. Systems Theory 2 (1968), 179-190.

5. P. R. Halmos and J. von Neumann, Operator methods in classical mechanics. II, Ann. of Math. (2) 43 (1942), 332-350.

Oregon State University, Corvallis, Oregon 97331 